\title{
A review of production technologies and materials for manufacturing of cardiovascular stents
}

\author{
Polanec, B. ${ }^{\text {a }, ~ K r a m b e r g e r, ~ J . ~}{ }^{a}$, Glodež, S. ${ }^{\text {a, }}$ \\ ${ }^{a}$ University of Maribor, Faculty of Mechanical Engineering, Maribor, Slovenia
}

\begin{abstract}
A B S T R A C T
The purpose of this article is to give a general overview of the production technologies of stents with consideration of their design and materials. Since the beginning of the use of stents in medicine for atherosclerosis treatment, their development has changed rapidly. Various stents have also been developed with the development of materials science, treatment techniques and new manufacturing processes. In this way the development has shifted from the initial bare-metal stents (BMS), to drug-eluting stents (DES) and bioresorbable stents (BRS), which are made of biodegradable polymers or metals. Various studies agree that it will be necessary to further review the experimentally obtained material properties with analytical and numerical studies. Here, the computational modelling (Finite element analysis - FEA and Computational fluid dynamics - CFD) was found as a valuable tool when evaluating stent mechanics and optimizing stent design. The development of the stent manufacturing technologies has also changed and been supplemented over the years. Nowadays, 3D printing could be an exciting manufacturing method to produce polymeric bio-materials, suitable for the latest generation of biodegradable stents applications.
\end{abstract}

(C) 2020 CPE, University of Maribor. All rights reserved.

\author{
ARTICLE INFO \\ Keywords: \\ Stent; \\ Bare-metal stent; \\ Drug-eluting stent; \\ Bio-resorbable stent; \\ Stent coatings; \\ Drug delivery; \\ Stent manufacturing: \\ Stent material; \\ Laser cutting; \\ Additive manufacturing (3D print- \\ ing) \\ *Corresponding author: \\ srecko.glodez@um.si \\ (Glodež, S.)
}

Article history:

Received 22 September 2020

Revised 8 December 2020

Accepted 12 December 2020

\section{References}

[1] Fogarotto, F. (2010). Finite element analysis of coronary artery stenting, Università degli Studi di Pavia Facoltà di Ingegneria, Pavia, Italy, from http://www-2.unipv.it/compmech/dissertations/fogarotto.pdf accessed July 2020.

[2] McCormic, C. (2018). 1 - Overview of cardiovascular stent designs, In: Wall, J.G., Podbielska, H., Wawrzyńska, M. (eds.), Finite element analysis of coronary artery stenting, Elsevier, Duxford, UK, 3-26, doi: 10.1016/B978-0-08100496-8.00001-9.

[3] Sigwart, U., Puel, J., Mirkovitch, V., Joffre, F., Kappenberger, L. (1987). Intravascular stents to prevent occlusion and re-stenosis after transluminal angioplasty, The New England Journal of Medicine, Vol. 316, No. 12, 701-706, doi: 10.1056/NEJM198703193161201.

[4] Rogers, C., Edelman, E.R. (1995). Endovascular stent design dictates experimental restenosis and thrombosis, Circulation, Vol. 91, No. 12, 2995-3001, doi:10.1161/01.CIR.91.12.2995.

[5] Schwartz, R.S., Chronos, N.A., Virmani, R. (2004). Preclinical restenosis models and drug-eluting stents: still important, still much to learn, Journal of the American College of Cardiology, Vol. 44, No. 7, 1373-1385, doi: 10.1016/i.jacc.2004.04.060.

[6] Morton, A.C., Crossman, D., Gunn, J. (2004). The influence of physical stent parameters upon restenosis, Pathologie Biologie, Vol. 52, No. 4, 196-205, doi: 10.1016/j.patbio.2004.03.013.

[7] Pache, J., Kastrati, A., Mehilli, J., Schühlen, H., Dotzer, F., Hausleiter, J., Fleckenstein, M., Neumann F.-J., Sattelberger, U., Schmitt, C., Müller, M., Dirschinger, J., Schömig, A. (2003). Intracoronary stenting and angiographic results. Strut thickness effect on restenosis outcome (ISAR-STEREO-2) trial, Journal of the American College of Cardiology, Vol. 41, No. 8, 1283-1288, doi: 10.1016/S0735-1097(03)00119-0. 
[8] Jayendiran, R., Nour, B., Ruimi, A. (2018). Fluid-structure interaction (FSI) analysis of stent-graft for aortic endovascular aneurysm repair (EVAR): Material and structural considerations, Material and structural considerations, Vol. 87, 95-110, doi: 10.1016/i.jmbbm. 2018.07.020.

[9] O'Brien, B., Carroll, W. (2009). The evolution of cardiovascular stent materials and surfaces in response to clinical drivers: A review, Acta Biomaterialia, Vol. 5, No. 4, 945-958, doi: 10.1016/i.actbio.2008.11.012.

[10] Bertrand, O.F., Sipehia, R., Mongrain, R., Rodés, J., Tardif, J.-C., Bilodeau, L., Côté, G., Bourassa, M.G. (1998). Biocompatibility aspects of new stent technology, Journal of the American College of Cardiology, Vol. 32, No. 3, 562571, doi: 10.1016/S0735-1097(98)00289-7.

[11] Mani, G., Feldman, M.D., Patel, D., Agrawal, C.M. (2007). Coronary stents: A materials perspective, Biomaterials, Vol. 28, No. 9, 1689-1710, doi: 10.1016/j.biomaterials.2006.11.042.

[12] Pache, J., Dibra, A., Mehilli, J., Dirschinger, J., Schömig, A., Kastrati, A. (2005). Drug-eluting stents compared with thin-strut bare stents for the reduction of restenosis: A prospective, randomized trial, European Heart Journal, Vol. 26, No. 13, 1262-1268, doi: 10.1093/eurheartj/ehi098.

[13] Htay, T., Liu, M.W. (2005). Drug-eluting stent: A review and update, Vascular Health and Risk Management, Vol. 1, No. 4, 263-276, doi: 10.2147/vhrm.2005.1.4.263.

[14] Yang, C., Burt, H.M. (2006). Drug-eluting stents: factors governing local pharmacokinetics, Advanced Drug Delivery Reviews, Vol. 58, No. 3, 402-411, doi: 10.1016/i.addr.2006.01.017.

[15] Sotomi, Y., Onuma, Y., Collet, C., Tenekecioglu, E., Virmani, R., Kleiman, N.S., Serruys, P.W. (2017). Bioresorbable scaffold: the emerging reality and future directions, Circulation Research, Vol. 120, No. 8, 1341-1352, doi: 10.1161/CIRCRESAHA.117.310275.

[16] Venkatraman, S., Boey, F. (2007). Release profiles in drug-eluting stents: Issues and uncertainties, Journal of Controlled Release, Vol. 120, No. 3, 149-160, doi: 10.1016/j.jconrel.2007.04.022.

[17] Commandeur, S., van Beusekom, H.M.M., van der Giessen, W.J. (2006). Polymers, drug release, and drug-eluting stents, Journal of Interventional Cardiology, Vol.19, No. 6, 500-506, doi: 10.1111/j.1540-8183.2006.00198.x.

[18] Joner, M., Finn, A.V., Farb, A., Mont, E.K., Kolodgie, F.D., Ladich, E., Kutys, R., Skorija, K., Gold, H.K., Virmani, R. (2006). Pathology of drug-eluting stents in humans: Delayed healing and late thrombotic risk, Journal of the American College of Cardiology, Vol. 48, No. 1, 193-202, doi: 10.1016/j.jacc.2006.03.042.

[19] Garcia-Touchard, A., Burke, S.E., Toner, J.L., Cromack, K., Schwartz, R.S. (2006). Zotarolimus-eluting stents reduce experimental coronary artery neointimal hyperplasia after 4 weeks, European Heart Journal, Vol. 27, No. 8, 988993, doi: 10.1093/eurheartj/ehi752.

[20] Brugaletta, S., Burzotta, F., Sabaté, M. (2009). Zotarolimus for the treatment of coronary artery disease: Pathophysiology, DES design, clinical evaluation and future perspective, Expert Opinion on Pharmacotherapy, Vol. 10, No. 6, 1047-1058, doi: 10.1517/14656560902837998.

[21] Byrne, R.A., Joner, M., Kastrati, A. (2009). Polymer coatings and delayed arterial healing following drug-eluting stent implantation, Minerva Cardioangiologica, Vol. 57, No. 5, 567-584.

[22] Finkelstein, A., McClean, D., Kar, S., Takizawa, K., Varghese, K., Baek, N., Park, K., Fishbein, M.C., Makkar, R., Litvack, F., Eigler, N.L. (2003). Local drug delivery via a coronary stent with programmable release pharmacokinetics, Circulation, Vol. 107 No. 5, 777-784, doi: 10.1161/01.CIR.0000050367.65079.71.

[23] Alexis, F., Venkatraman, S.S., Rath, S.K., Boey, F. (2004). In vitro study of release mechanisms of paclitaxel and rapamycin from drug-incorporated biodegradable stent matrices, Journal of Controlled Release, Vol. 98, No. 1, 6774, doi: 10.1016/j.jconrel.2004.04.011.

[24] Falotico, R., Parker, T., Grishaber, R., Price, S., Cohen, S.A., Rogers, C. (2009). NEVOTM: A new generation of sirolimus-eluting coronary stent, EuroIntervention, Vol. 5 (Supplement F), F88-F93.

[25] Stefanini, G.G., Byrne, R.A., Serruys, P.W., de Waha, A., Meier, B., Massberg, S., Jüni, P., Schömig, A., Windecker, S., Kastrati, A. (2012). Biodegradable polymer drug-eluting stents reduce the risk of stent thrombosis at 4 years in patients undergoing percutaneous coronary intervention: A pooled analysis of individual patient data from the ISAR-TEST 3, ISAR-TEST 4, and LEADERS randomized trials, European Heart Journal, Vol. 33, No. 10, 1214-1222, doi: 10.1093 /eurhearti/ehs086.

[26] O'Brien, B., Zafar, H., Ibrahim, A., Zafar, J., Sharif, F. (2016). Coronary stent materials and coatings: A technology and performance update, Annals of Biomedical Engineering, Vol. 44, No. 2, 523-535, doi: 10.1007/s10439-0151380-x.

[27] Wessely, R., Hausleiter, J., Michaelis, C., Jaschke, B., Vogeser, M., Milz, S., Behnisch, B., Schratzenstaller, T., RenkeGluszko, M., Stöver, E., Wintermantel, E., Kastrati, A., Schömig, A. (2005). Inhibition of neointima formation by a novel drug-eluting stent system that allows for dose-adjustable, multiple, and on-site stent coating, Arteriosclerosis, Thrombosis, and Vascular Biology, Vol. 25, No. 4, 748-753, doi: 10.1161/01.ATV.0000157579.52566.ee.

[28] Dibra, A., Kastrati, A., Mehilli, J., Pache, J., von Oepen, R., Dirschinger, J., Schömig, A. (2005). Influence of stent surface topography on the outcomes of patients undergoing coronary stenting: A randomized double-blind controlled trial, Catheterization \& Cardiovascular Interventions, Vol. 65, No. 3, 374-380, doi: 10.1002/ccd.20400.

[29] Demidov, V., Currie, D., Wen, J. (2017). Patent watch: Patent insight into polymer-free drug-eluting stents, Nature Reviews Drug Discovery, Vol. 16, No. 4, 230-231, doi: 10.1038/nrd.2017.32.

[30] Schwartz, R.S., Chronos, N.A., Virmani, R. (2004). Preclinical restenosis models and drug-eluting stents: Still important, still much to learn, Journal of the American College of Cardiology, Vol. 44, No. 7, 1373-1385, doi: 10.1016/i.jacc.2004.04.060.

[31] Ormiston, J.A., Serruys, P.W., Regar E., Dudek, D., Thuesen, L., Webster, M.W.I., Onuma, Y., Garcia-Garcia, H.M., McGreevy, R. (2008). A bioabsorbable everolimus-eluting coronary stent system for patients with single de-novo coronary artery lesions (ABSORB): A prospective open-label trial, The Lancet, Vol. 371, No. 9616, 899-907, doi: $10.1016 / \mathrm{S} 0140-6736(08) 60415-8$. 
[32] Onuma, Y., Serruys, P.W., Perkins, L.E.L., Okamura, T., Gonzalo, N., García-García, H.M., Regar, E., Kamberi, M., Powers, J.C., Rapoza, R., van Beusekom, H., van der Giessen, W., Virmani, R. (2010). Intracoronary optical coherence tomography and histology at 1 month and 2, 3, and 4 years after implantation of everolimus-eluting bioresorbable vascular scaffolds in a porcine coronary artery model: An attempt to decipher the human optical coherence tomography images in the ABSORB trial, Circulation, Vol. 122, No. 22, 2288-2300, doi: 10.1161/ CIRCULATIONAHA.109.921528.

[33] Alexy, R.D., Levi, D.S. (2013). Materials and manufacturing technologies available for production of a pediatric bioabsorbable stent, BioMed Research International, Vol. 2013, Article ID 137985, doi: 10.1155/2013/137985.

[34] Beshchasna, N., Saqib, M., Kraskiewicz, H., Wasyluk, Ł., Kuzmin, O., Duta, O.C., Ficai, D., Ghizdavet, Z., Marin, A., Ficai, A., Sun, Z., Pichugin, V.F., Opitz, J., Andronescu, E. (2020). Recent advances in manufacturing innovative stents, Pharmaceutics, Vol. 12, No. 4, 349, doi: /10.3390/pharmaceutics12040349.

[35] Peuster, M., Wohlsein, P., Brügmann, M., Ehlerding, M., Seidler, K., Fink, C., Brauer, H., Fischer, A., Hausdorf, G. (2001). A novel approach to temporary stenting: Degradable cardiovascular stents produced from corrodible metal - results 6-18 months after implantation into New Zealand white rabbits, Heart, Vol. 86, No. 5, 563-569, doi: $10.1136 /$ heart.86.5.563.

[36] Huang, T., Cheng, J., Zheng, Y.F. (2014). In vitro degradation and biocompatibility of Fe-Pd and Fe-Pt composites fabricated by spark plasma sintering, Material Science and Engineering: C, Vol. 35, 43-53, doi: 10.1016/i.msec. 2013.10.023.

[37] Bowen, P.K., Drelich, J., Goldman, J. (2013). Zinc exhibits ideal physiological corrosion behavior for bioabsorbable stents, Advanced Materials, Vol. 25. No. 18, 2577-2582, doi: 10.1002/adma.201300226.

[38] Pierson, D., Edick, J., Tauscher, A., Pokorney, E., Bowen, P., Gelbaugh, J., Stinson, J., Getty, H., Lee, C.H., Drelich, J., Goldman, J. (2012). A simplified in vivo approach for evaluating the bioabsorbable behavior of candidate stent materials, Journal Biomedicine Materials and Research, Vol. 100B, No. 1, 58-67, doi: 10.1002/jbm.b.31922.

[39] Erbel, R., Di Mario, C., Bartunek, J., Bonnier, J., de Bruyne, B., Eberli, F., Erne, P., Haude, M., Heublein, B., Horringan, M., Ilsley, C., Böse D., Koolen, J., Lüscher, T.F., Weissman, N., Waksman, R. (2007). Temporary scaffolding of coronary arteries with bioabsorbable magnesium stents: a prospective, non-randomised multicentre trial, The Lancet, Vol. 369, No. 9576, 1869-1875, doi: 10.1016/S0140-6736(07)60853-8.

[40] Su, Y., Cockerill, I., Wang, Y., Qin, Y.-X., Chang, L., Zheng, Y., Zhu, D. (2019). Zinc-based biomaterials for regeneration and therapy, Trends in Biotechnology, Vol. 37, No. 4, 428-441, doi: 10.1016/i.tibtech.2018.10.009.

[41] Yang, H., Wang, C., Liu, C., Chen, H., Wu, Y., Han, J., Jia, Z., Lin, W., Zhang, D., Li, W., Yuan, W., Guo, H., Li, H., Yang, G., Kong, D., Zhu, D., Takashima, K., Ruan, L., Nie, J., Li, X., Zheng, Y. (2017). Evolution of the degradation mechanism of pure zinc stent in the one-year study of rabbit abdominal aorta model, Biomaterials, Vol. 145, 92-105, doi: 10.1016/i.biomaterials.2017.08.022

[42] Bowen, P.K., Shearier, E.R., Zhao, S., Guillory 2nd, R.J., Zhao, F., Goldman, J., Drelich, J.W. (2016). Biodegradable metals for cardiovascular stents: From clinical concerns to recent Zn-alloys, Advanced Healthare Materials, Vol. 5, No. 10, 1121-1140, doi: 10.1002/adhm.201501019.

[43] Zhu, D., Su, Y., Zheng, Y., Fu, B., Tang, L., Qin, Y.-X. (2018). Zinc regulates vascular endothelial cell activity through zinc-sensing receptor ZnR/GPR39, American Journal of Physiology - Cell Physiology, Vol. 314, No. 4, C404-C414, doi: 10.1152/ajpcell.00279.2017.

[44] Lei, Y., Chen, X., Li, Z., Zhang, L., Sun, W., Li, L., Tang, F. (2020). A new process for customized patient-specific aortic stent graft using 3D printing technique, Medical Engineering and Physics, Vol. 77, 80-87, doi: 10.1016/ j.medengphy.2019.12.002.

[45] Yang, L., Chen, X., Zhang, L., Li, L., Kang, S., Wang, C., Sun, W. (2019). Additive manufacturing in vascular stent fabrication, MATEC Web of Conf., 2018 International Conference on Materials Science and Manufacturing Engineering, Vol. 253, Article number 03003, doi: 10.1051/matecconf/201925303003.

[46] Zhang, L., Chen, X., Liu, M. (2017). Research of customized aortic stent graft manufacture, IOP Conference Series: Materials Science and Engineering, Vol. 187, 012027, doi: 10.1088/1757-899x/187/1/012027.

[47] Huang, B., Gale, D.C., Gale, Hossainy, S.F.A. (2011). Fabricating polymer stents with injection molding, Patent Application Publication, No. US 2011/0169197 A1.

[48] Guerra, A.J., Ciurana, J. (2018). Stent's manufacturing field: Past, present, and future prospects, In: Angiography, Amukçu, B. (ed.), IntechOpen, 41-60, doi: 10.5772/intechopen.81668.

[49] Flege, C., Vogt, F., Höges, S., Jauer, L., Borinski, M., Schulte, V.A., Hoffmann, R., Poprawe, R., Meiners, W., Jobmann, M., Wissenbach, K., Blindt, R. (2013). Development and characterization of a coronary polylactic acid stent prototype generated by selective laser melting, Journal of Materials Science: Materials in Medicine, Vol. 24, No. 1, 241255, doi: $10.1007 / \mathrm{s} 10856-012-4779-\mathrm{z}$.

[50] Finazzi, V., Demir, A.G., Biffi, C.A., Chiastra, C., Migliavacca, F., Petrini, L., Previtali, B. (2019). Design rules for producing cardiovascular stents by selective laser melting: Geometrical constraints and opportunities, Procedia Structural Integrity. Vol. 15, 16-23, doi: 10.1016/j.prostr.2019.07.004.

[51] Park, S.A., Lee, S.J., Lim, K.S., Bae, I.H., Lee, J.H., Kim, W.D., Jeon, M.H., Park, J.-K. (2015). In vivo evaluation and characterization of a bio-absorbable drug-coated stent fabricated using a 3D-printing system, Materials Letters, Vol. 141, 355-358, doi: 10.1016/i.matlet.2014.11.119.

[52] Tumbleston, J.R., Shirvanyants, D., Ermoshkin, N., Janusziewicz, R., Johnson, A.R., Kelly, D., Chen, K., Pinschmidt, R., Rolland, J.P., Ermoshkin, A., Samulski, E.T., DeSimone, J.M. (2015). Additive manufacturing. Continuous liquid interface production of 3D objects, Science, Vol. 347, No. 6228, 1349-1352 \& doi: 10.1126/science.aaa2397.

[53] van Lith, R., Baker, E., Ware, H., Yang, J., Farsheed, A.C., Sun, C., Ameer, G. (2017). 3D-printing strong highresolution antioxidant bioresorbable vascular stents, Advanced Materials Technologies, Vol. 1, No. 9, doi: $\underline{10.1002 / \mathrm{admt} .201600138}$ 
[54] Ware, H.O.T., Farsheed, A.C., van Lith, R., Baker, E., Ameer, G., Sun. C. (2017). Process development for highresolution 3D-printing of bioresorbable vascular stents, In: Proceedings Volume 10115, Advanced Fabrication Technologies for Micro/Nano Optics and Photonics X, SPIE OPTO, 2017, San Francisco, California, USA, doi: $10.1117 / 12.2252856$.

[55] Cabrera, M.S., Sanders, B., Goor, O.J.G.M., Driessen-Mol, A., Oomens, C.W.J., Baaijens, F.P.T. (2017). Computationally designed 3D printed self-expandable polymer stents with biodegradation capacity for minimally invasive heart valve implantation: A proof-of-concept study, 3D Printing and Additive Manufacturing, Vol. 4, No. 1, 19-29, doi: $10.1089 / 3 \mathrm{dp} .2016 .0052$.

[56] Guerra, A.J., Ciurana, J. (2017). 3D-printed bioabsordable polycaprolactone stent: The effect of process parameters on its physical features, Materials \& Design, Vol. 137, 430-437, doi: 10.1016/i.matdes.2017.10.045.

[57] Guerra, A., Roca, A., de Ciurana, J. (2017). A novel 3D additive manufacturing machine to biodegradable stents, Procedia Manufacturing, Vol. 13, 718-723, doi: 10.1016/i.promfg.2017.09.118. 
APEM
Advances in Production Engineering \& Management Letnik 15 | Številka 4 | December 2020 | Strani 390-402 https://doi.org/10.14743/apem2020.4.373
ISSN 1854-6250

Spletna stran: apem-journal.org Izvirni znanstveni članek

\title{
Pregled proizvodnih tehnologij in materialov za izdelavo žilnih opornic
}

\author{
Polanec, B. ${ }^{a}$, Kramberger, J. ${ }^{a}$, Glodež, S. ${ }^{\text {a }}{ }^{*}$ \\ aUniversity of Maribor, Faculty of Mechanical Engineering, Maribor, Slovenia
}

\section{POVZETEK}

Namen tega članka je podati splošen pregled tehnologij za izdelavo žilnih opornic z upoštevanjem njihove zasnove in materialov. Od začetka uporabe žilnih opornic v zdravstvu, ko so bile uporabljene za zdravljenje ateroskleroze, se je njihov razvoj hitro spremenil. Z razvojem različnih vej znanosti, kot sta znanost o materialih in znanost o obdelovalnih tehnikah in proizvodnih procesih, so se razvile tudi različne opornice. Na ta način se je razvoj premaknil od začetnih kovinskih opornic brez prevleke (BMS), do opornic, ki izločajo zdravila (DES) in biorazgradljivih opornic (BRS), ki so izdelane iz biološko razgradljivih polimerov ali kovin. Številne študije poudarjajo, da bo treba eksperimentalno pridobljene lastnosti materialov podpreti tudi $\mathrm{z}$ analitičnimi in numeričnimi študijami. Izkazalo se je, da je računalniško modeliranje (npr. analiza končnih elementov - FEA ter računalniška dinamika tekočin - CFD) dragoceno orodje pri ocenjevanju mehanike opornic in pri optimizaciji njihove zasnove. $\mathrm{Z}$ leti so se spreminjale in dopolnjevale tudi tehnologije izdelave opornic. 3D-tiskanje je danes najbolj obetavna metoda za proizvodnjo polimernih biomaterialov, primernih za najnovejšo generacijo biorazgradljivih opornic.

(C) 2020 CPE, University of Maribor. All rights reserved.

\section{PODATKI O ČLANKU}

Ključne besede:

Opornica;

Kovinska opornica brez prevleke; Opornica, ki izloča zdravila;

Biorazgradljiva opornica;

Prevleke za opornice;

Dostava zdravil;

Izdelava opornic;

Material za opornice;

Lasersko rezanje;

Dodajalna izdelava (3D-tiskanje)

*Kontaktna oseba:

srecko.glodez@um.si

(Glodež, S.)

Zgodovina članka:

Prejet 22. septembra 2020

Popravljen 8. decembra 2020

Sprejet 12. decembra 2020 